\title{
Metasurface for Reciprocal Spin-Orbit Coupling of Light on Waveguiding Structures
}

\author{
Quanbo Jiang, ${ }^{1,2, *}$ Julien Laverdant, ${ }^{1}$ Clementine Symonds, ${ }^{1}$ Aline Pham,${ }^{2}$ Cecile Leluyer, ${ }^{1}$ \\ Stephan Guy, ${ }^{1}$ Aurelien Drezet, ${ }^{2}$ and Joel Bellessa ${ }^{1, \dagger}$ \\ ${ }^{1}$ Institut Lumière Matière, CNRS, Université Claude Bernard Lyon 1, F-69622 Villeurbanne, France \\ ${ }^{2}$ Institut NEEL, CNRS, Université Grenoble Alpes, F-38042 Grenoble, France
}

(Received 22 October 2017; revised manuscript received 15 March 2018; published 17 July 2018)

\begin{abstract}
Light manipulation through spin-orbit coupling opens new perspectives in photonics and particularly in integrated optics. The reverse spin Hall effect, where the guided wave direction affects the polarization properties of the light scattered by a nanostructure, is a key effect for the development of new functionalities at the connection between integrated structures and free space, and could find application in chiral quantum optics, modulation, and multiplexing. We show that metasurfaces represent a promising platform for the reverse spin Hall effect. Using a periodic array of $\Lambda$-shaped metallic nanoantenna, we control the polarization of the extracted light with the guided wave-propagation direction, but also the number of output directions and the polarization of each one. These results and the versatility of metasurfaces for the spin Hall effect could be extended to various frequencies and materials, such as silicon photonics at telecom wavelength.
\end{abstract}

DOI: 10.1103/PhysRevApplied.10.014014

\section{INTRODUCTION}

Spin-orbit coupling of light [1,2] has been widely investigated as a way to control light propagation, with its spin. The spin Hall (SH) effect [3-6], which couples the internal momentum of light to its orbital angular momentum, can be applied to tune the scattering of a nanoparticle towards a confined propagating wave [7]. This type of $\mathrm{SH}$ effect has been reported for surface plasmon polaritons and guided waves [7-11]. The spin manipulation of guided modes is of particular interest for integrated optics [12], quantum optics [13], optoelectronics, biosensors [14-16]. The reverse SH effect, where the direction of the guided wave affects the spin of the light scattered by a nanostructure [17], is also of particular interest for controlling the decoupling of guided modes into free space, in a polarization-dependent way. The first demonstration of reverse SH effect was performed in 2014 with a metallic nanoparticle and surface plasmons [7] at the same time as the demonstration of polarization control by a single nanoantenna $[18,19]$.

The exploitation of metasurfaces, i.e., periodic arrays of subwavelength nanoparticles, offers a promising platform for the spin Hall effect in the sense that their

*quanbo.jiang@cea.fr

†joel.bellessa@univ-lyon1.fr intrinsic optical properties offer large degrees of freedom, allowing a wide variety of functionalities. Spin-dependent dispersion of spontaneous [20] or stimulated [21] emission has been demonstrated with metasurfaces. In the case of the reverse SH effect, the metasurface geometries, i.e., organization and shape of the nanoparticles, could lead to a more accurate control of the scattering and emission polarization compared with isolated nanoparticles [22].

In this work, we evidence a reciprocal effect of spinorbit coupling with a metasurface on a slab waveguide. The metasurface constituted by a periodic collection of $\Lambda$-shaped nanoantennas (NAs) is used for extracting the guided light out of the waveguide. We observe that the polarization states of the decoupled light in the free space strongly depend on the propagation direction in the waveguide, and also on the extraction angle. By selecting an adequate extraction direction, it is possible to generate linear, left- or right-handed circular polarized light. An analytical model taking into account the antenna shape and periodicity is implemented, and agrees with the experiments. Finally, we theoretically show the versatility of the metamaterials for the reverse spin Hall effect in giving an example of an unidirectional circular light extractor for a waveguide, with a polarization associated to the propagating direction. Such a device could be applied to integrated optics such as a demultiplexer, demodulation, quantum information and processing [23-25]. 


\section{SAMPLE DESIGN AND EXPERIMENTAL SETUP}

The metasurface used to evidence reciprocal SH coupling is constituted by an array of $\Lambda$-shaped gold antennas, fabricated on a $\mathrm{TiO}_{2}$ waveguide by electron-beam lithography (see the Appendix). The whole metasurface consists in an array of $4 \times 4$ antennas with a period $p=650 \mathrm{~nm}$ in both directions. Each $\Lambda$-shaped antenna is formed by two gold ridges of length $L=200 \mathrm{~nm}$ and width $w=50 \mathrm{~nm}$. The apex angle between both ridges is $90^{\circ}$, which leads to a center-to-center distance of $D=160 \mathrm{~nm}$. The layout of the antenna array and a scanning electron microscope (SEM) image are shown in Figs. 1(a) and 1(b). The $\Lambda$ shaped geometry has been designed to induce a phase difference between both arms of the antenna when illuminated by a guided wave propagating in the $x$ direction. The re-emission of dephased perpendicular dipoles associated with both antenna arms is the key to the spin selection of the light scattered in free space. The effect of the individual antenna shape combines with the periodicity of the metasurface to allow an efficient control of the guided mode decoupling in direction and polarization.

In order to couple the incident light into the guided mode, metallic gratings are deposited on the surface of the waveguide, $10 \mu \mathrm{m}$ away from both sides of the metasurface. The modulation period has been chosen as $a=\lambda / n_{\text {eff }}$. A period of $a=360 \mathrm{~nm}$ allows an efficient coupling at 633 $\mathrm{nm}$. An optical image of the sample illuminated in white light is presented in Fig. 1(d) with the NA array at the center and the couplers on both sides.

To measure the scattering of the NA array, we use a microscopy setup with an immersion microscope objective (Numerical aperture (N.A.) 1.49), presented in Fig. 1(f). The sample is excited on the coupler through the glass substrate with a laser light at $633 \mathrm{~nm}$ with a beamwaist diameter of $1 \mu \mathrm{m}$, and the propagation is detected in backscattering. Fourier space imaging providing the angular dispersion of the radiated emission [26] can be performed, as well as direct imaging of the surface. Beam blocks in the intermediate plane of the direct or Fourier space are also used to select the emission from specific angle or position. The different images are recorded in linear or circular polarized light.

In order to have a complete description of the polarization state of the light, the Stokes parameters have also been determined. For this purpose, a rotating quarter-wave plate (QWP) followed by a fixed linear polarizer is introduced into the pathway of the light. The polarization state
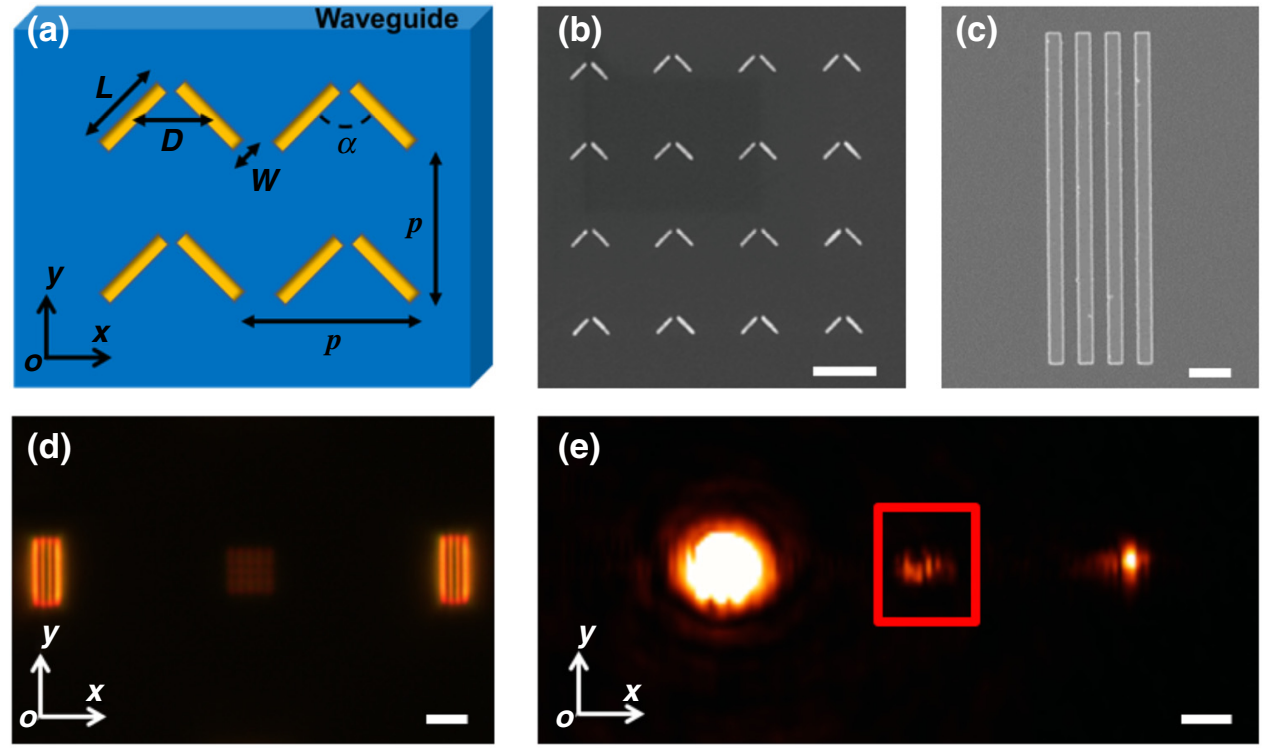

(f)

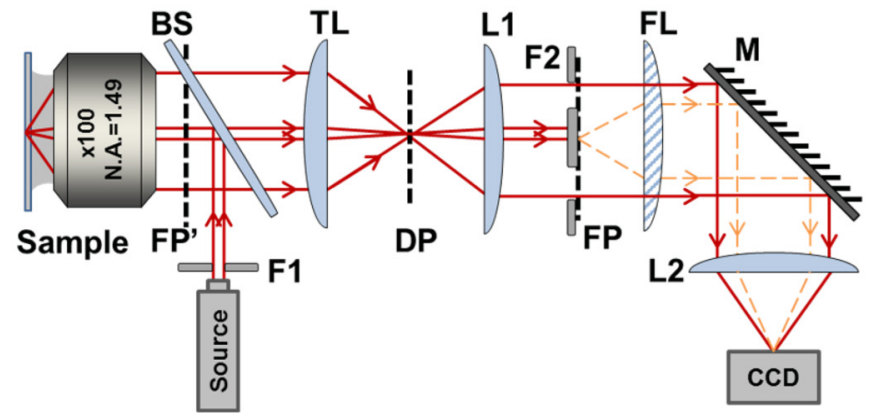

FIG. 1. (a) Layout of the $\Lambda$ shaped antenna array. (b),(c) SEM images of the $\Lambda$-shaped antenna array and the coupler with 500$\mathrm{nm}$ scale bars, respectively. (d) Optical image in reflection of the sample with a $5-\mu \mathrm{m}$ scale bar. The NA array is the square at the center of the image. (e) Image of the light scattered by the sample with a $5-\mu \mathrm{m}$ scale bar. The laser $(\lambda=633 \mathrm{~nm})$ is injected in the waveguide through the left coupler, corresponding to the bright spot on the image. The red box shows the region of the antenna array. (f) Experimental setup for a direct imaging of the sample. F2 is a beam block used for the direct imaging of the scattered light. When the lens FL is added, an image of the Fourier plane (FP) is obtained on the detector and the corresponding rays are shown as dashed lines. 
of each pixel of the image is deduced from the measurement of intensity images for different QWP angles (see the Appendix). From the Stokes parameters, we deduce the overall intensity, the polarization ratio, and the polarization ellipticity.

\section{RECIPROCAL EFFECT OF SPIN-ORBIT COUPLING}

A guided wave is generated by focusing a laser spot on the left metallic grating. The resulting image is shown in Fig. 1(e). Three spots can be seen on the image associated with the propagation in the waveguide; the brighter one corresponds to the laser excitation, the weak spot in the center to the nanoantenna array, and the spot on the right is associated with the second metallic coupler. As the guided mode is not coupled to free-space radiation, no light is observed on the unstructured waveguide region. The light extracted from the NA array does not present a well-defined polarization, i.e., no intensity variation can be seen by selecting linear or circular polarization in the image.

To more thoroughly understand the light scattered by the NA, the diffusion of the NA array as a function of the scattering direction is studied by imaging the Fourier plane, with an excitation coming from the left coupler. In order to select a $k$ space image only related to the light coming from the NA array, and exclude the coupler scattering, a diaphragm is inserted into a direct intermediate plane (the red square in Fig. 1(e) represents the selected region). The light polarization at the position of the NA array as a function of the scattering direction is shown in Fig. 2. The color in the image corresponds to the intensity of polarized light (which is nearly equal to the total intensity) and the polarization is represented by ellipses. Nine bright spots can be seen in this image, corresponding to the array diffraction orders. The striking point in this $k$ space image lies in the polarization associated with the different diffraction directions: on the left and on the right of the image, a polarization with a strong ellipticity and with opposite rotation signs can be observed. These different polarization contributions of the scattering explain clearly why a defined polarization cannot be measured without angle selection in direct images as in Fig. 1(e). Polarization fluctuations are presented within the different spots in Fig. 2. These variations could be attributed to the laser light scattered by the coupler, which partially remains after the spatial filtering. This nonuniform stray intensity is more sensitive on the side of the spot where the signal intensity is lower.

To obtain a polarization handedness of the extracted light, which changes with the direction of the guided wave, it is thus necessary to select given regions of the Fourier plane, i.e., scattering angles. We have measured direct images similar to the images in Fig. 1(e), but with restrained diffraction directions. For this purpose, a diaphragm is now inserted into an intermediate Fourier plane, and the direct image of the sample surface is imaged on the camera. The resulting direct images, with the right part of the Fourier plane selected, and recorded with a circular polarization are presented in Fig. 3. When the excitation is on the left coupler, corresponding to a wave arriving from the left on the NA array, the diffusion can only be seen in left handed polarization (LHP); no light can be seen when the detection is right handed polarization (RHP). When the excitation is on the right, the diffused light is RHP. These measurements in direct space agree with the polarization of the $k$ space images. It is thus possible to control the polarization of the decoupling of a guided wave, and a quasi-perfect reciprocal SH effect can be achieved for $\Lambda$-shaped nanoantennas by selecting given scattering directions. To give a quantitative estimation of the polarization control, we introduce a factor characterizing the direction selectivity as a function of the circular

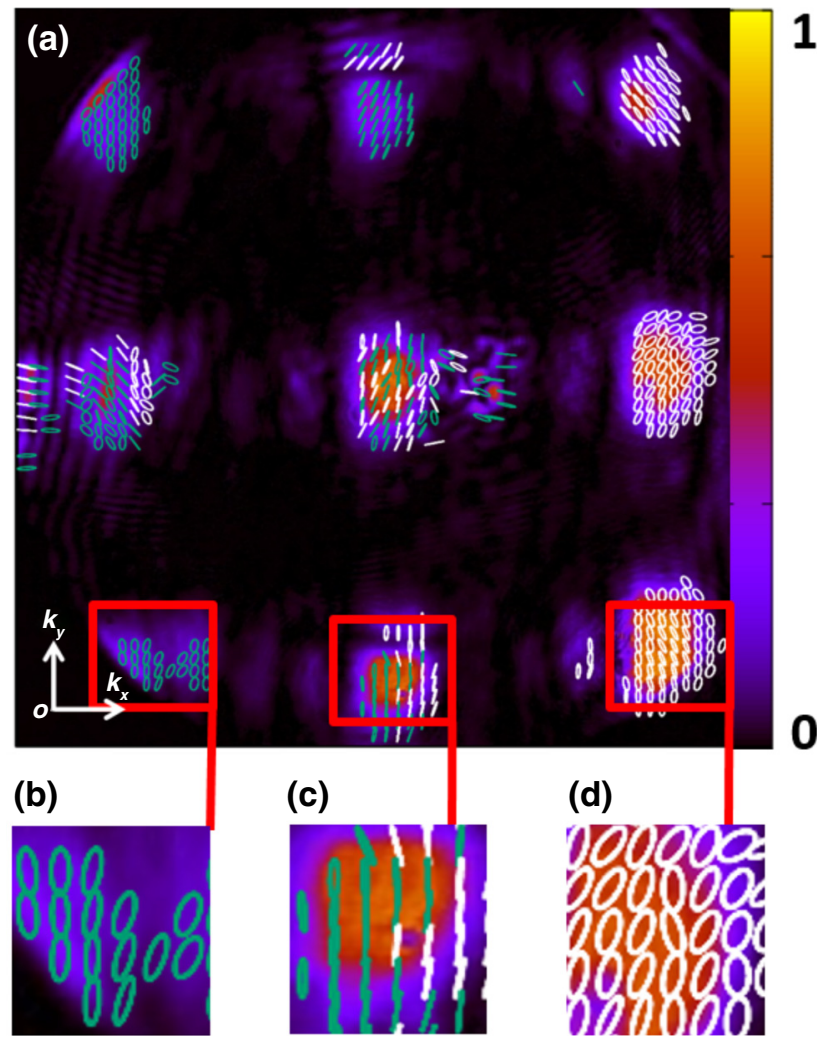

FIG. 2. (a) Polarization analysis of the FP image for an excitation coming from the left of the metasurface. The nine spots correspond to different diffraction orders. The color of the ellipses (green and white) represents the left- or right-hand type of elliptical polarization. (b)-(d) Enlarged images of the polarization states in the red box, indicating that the polarization states on the left and right sides have opposite handedness and the polarization in the middle is linear. 

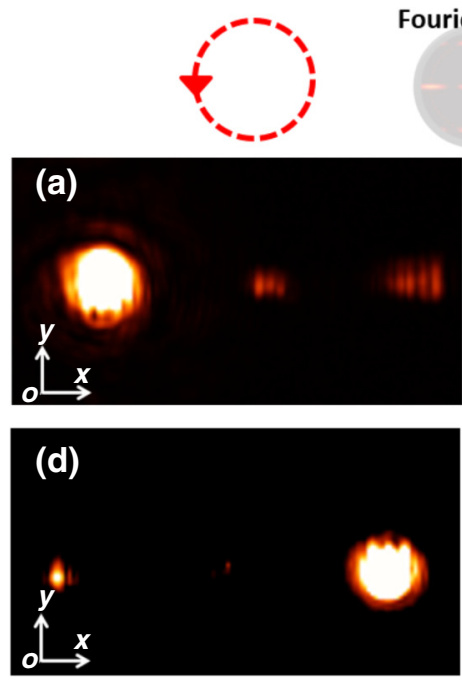

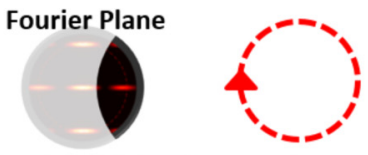

(b)
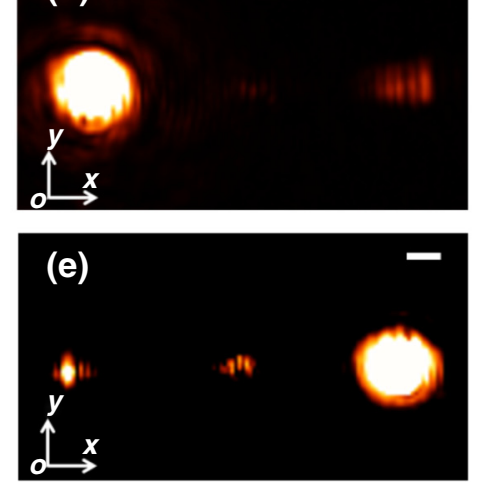

(c)

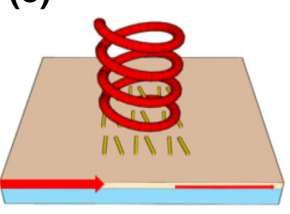

(f)

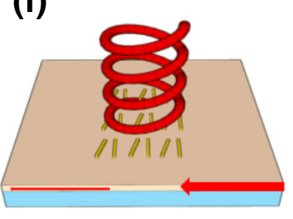

FIG. 3. Reciprocal effect on the direct images. Diffraction order is selected by choosing the right part of the Fourier plane. (a),(b) The laser is focused on the left coupler with LHP (a) and RHP (b) detection. (d),(e) Direct images with an excitation on the right coupler with LHP (d) and RHP (e) detection. (c),(f) illustrate the output polarization with different direction of guided waves: RHP when the guided wave comes from the right and LHP from the left. polarization detected:

$$
\begin{aligned}
& C_{\mathrm{LHP}}=\left(I_{\mathrm{LHP}}^{\text {right }}-I_{\mathrm{LHP}}^{\text {left }}\right) /\left(I_{\mathrm{LHP}}^{\text {right }}+I_{\mathrm{LHP}}^{\text {left }}\right), \\
& C_{\mathrm{RHP}}=\left(I_{\mathrm{RHP}}^{\text {right }}-I_{\mathrm{RHP}}^{\text {left }}\right) /\left(I_{\mathrm{RHP}}^{\text {right }}+I_{\mathrm{RHP}}^{\text {left }}\right),
\end{aligned}
$$

where $I_{\mathrm{LHP}}^{\text {right }}\left(I_{\mathrm{LHP}}^{\text {left }}\right)$ is the intensity detected in LHP coming from the right (left) coupler. These factors are equal to \pm 1 for perfect direction selectivity for a given polarization. By integrating the scattering on the metasurface for the four different cases, we find $C_{\mathrm{LHP}}=-0.87$ and $C_{\mathrm{RHP}}=0.86$, confirming the good direction selectivity observed on the images of Fig. 3.

Concerning the intensity measurement in Fig. 3(e), the fraction of light intensity detected on the NA array and on the decoupler are $2.1 \%$ and $7.5 \%$ with respect to the intensity detected on the excited coupler. The light intensity propagating in the waveguide can be deduced from the decoupler measurement. From our simulation of finite-difference time domain for the decoupler grating, the coupling efficiency to the waveguide is determined as $25 \%$. Thus, we can deduce the extraction efficiency of the antenna array as $7 \%$. This ratio could be improved by increasing the number of antennas in the array.

\section{ANALYTICAL MODEL FOR RECIPROCAL SPIN-ORBIT COUPLING}

To extract the physical parameters which influence the reverse SH effect, a simple analytical model will be presented first. We will first only consider the Fourier plane direction corresponding to the diffraction of the guided mode by an infinite array of antennas. In this case, according to the conservation of momentum, the wave vector of diffraction by the array should respect

$$
K_{n}=K_{g}+\frac{2 \pi}{p} n,
$$

where $K_{g}$ represents the wave vector of the guide mode given by $K_{g}=\left(2 \pi / \lambda_{0}\right) n_{\text {eff. }}$. The effective refractive index of waveguide $n_{\text {eff }}=1.75$ in transverse magnetic (TM) polarization. The integer number $n$ represents the order of diffraction and $p$ is the period of the array of $\Lambda$ shaped antennas. We limit our analysis to the diffraction orders, which can be detected by the microscope objective (N.A. $=1.49$ ). For a grating period $p$ fixed at $650 \mathrm{~nm}$, only the orders $n=-1,-2,-3$ can be observed, located, respectively, on the right side, the nearly center, and the left side of the Fourier image. It has to be noted that this approach would give the same results for a scattering in free vacuum space; surrounding the guided mode, the only modification accepted would be a maximum N.A. of 1 . We then consider the scattering of a single $\Lambda$-shaped antenna in the diffraction direction defined above. The scattering of both arms of the $\Lambda$-shaped antenna is modeled by two re-emitting dipoles, oriented along the length of the two ridges $\left[\widehat{n}_{1}\right.$ and $\widehat{n}_{2}$ indicated in Fig. 4(a)], excited by the propagating guided wave. The total electric field radiated by one antenna is given by

$$
\vec{E}(K) \propto\left[\left(\widehat{n}_{1} \cdot \overrightarrow{E_{g}}\right) \widehat{n}_{1} e^{-i \overrightarrow{K_{n}} \cdot \overrightarrow{x_{1}}}+\left(\widehat{n_{2}} \cdot \overrightarrow{E_{g}}\right) \widehat{n}_{2} e^{-i \overrightarrow{K_{n}} \cdot \overrightarrow{x_{2}}}\right],
$$

where $\overrightarrow{x_{1}}$ and $\overrightarrow{x_{2}}$ represent the position of the two dipoles, fixed at the center of the ridges, and $\overrightarrow{E_{g}}$ denotes the electric field of the guided wave. Its amplitude has been considered constant because the size of the metasurface is small compared to the guided mode propagation length and the losses due to the scattering are negligible. With the origin set in the middle of the $\Lambda$-shaped ridges, and a propagation along $\widehat{x}$, the total electric field is written as

$$
\begin{gathered}
\vec{E}(K) \propto(\widehat{x}-\widehat{y}) e^{i\left(K_{g}-K_{n}\right)(D / 2)} \\
+(\widehat{x}+\widehat{y}) e^{-i\left(K_{g}-K_{n}\right)(D / 2)}, \\
\propto(\widehat{x}-\widehat{y}) e^{i(n D / p) \pi}+(\widehat{x}+\widehat{y}) e^{-i(n D / p) \pi} .
\end{gathered}
$$


The polarization of the scattered light results from the phase difference of the re-emission of the orthogonally polarized dipoles (if equal to $\pi / 2$, a circular polarization is scattered). In our practical case, $D / p=\frac{1}{4}$, therefore, the total electric fields of three orders of diffraction are given by

$$
\begin{aligned}
& \vec{E}\left(K_{n=-1}\right) \propto \widehat{x}+\imath \widehat{y}, \\
& \vec{E}\left(K_{n=-2}\right) \propto \imath \widehat{y}, \\
& \vec{E}\left(K_{n=-3}\right) \propto \widehat{x}-\imath \widehat{y} .
\end{aligned}
$$

These three orders of diffraction correspond to the left $(n=-1)$, central $(n=-2)$, and right part $(n=-3)$ of the Fourier plane. The obtained results agree with the opposite circular polarization handedness measured experimentally for left and right parts of the Fourier image (Fig. 2) and the quasi linearly polarized central area in the measured image. Basically, the scattering directions are governed by the array symmetry and size, and the polarization repartition in these main directions by the nanoantenna geometry.

While the previous analytical approach emphasizes the main physics of the system, for quantitative comparison we develop a systematic numerical model taking into account the limited size of the array, which is then used to simulate the scattering of the nanoantennas in the Fourier plane of a high-NA microscope objective. This approach rigorously involves the nonparaxial light propagation and geometrical aberration in the microscope, which have to be considered for an accurate description of the imaging process $[27,28]$. To simulate the reversible SH effect for a metasurface formed by an array of $4 \times 4$ nanoantennas, we thus consider 16 pairs of orthogonal in-plane dipoles (oriented perpendicularly to each arm of the $\Lambda$-shaped antennas) and coherently sum their electric fields as a function of the imaging direction $[10,20,29]$. The interactions between each dipole are fairly neglected as assumed in [29]. The intensity and polarization associated with the total electric field is presented in Fig. 4. Bright spots corresponding to the main diffraction directions can be observed. The polarization in each spot is quasi uniform with a strong ellipticity and opposite handedness. The position of the intensity spot, as well as the polarization within each spot, is in qualitative agreement with the experimental image of Fig. 2.

Two parameters can be tuned quasi independently to adjust the scattering properties of the metasurfaces, from one side the period of the array and on the other side the pattern, here the $\Lambda$-shaped antennas. The periodic structure of the array and its extension affects the position and the width of the scattering spots in the Fourier plane. In order to obtain a single spot in the Fourier plane, the period of the array is reduced to bring the order -1 in the center. The scattering is thus perpendicular to the surface,

(a)

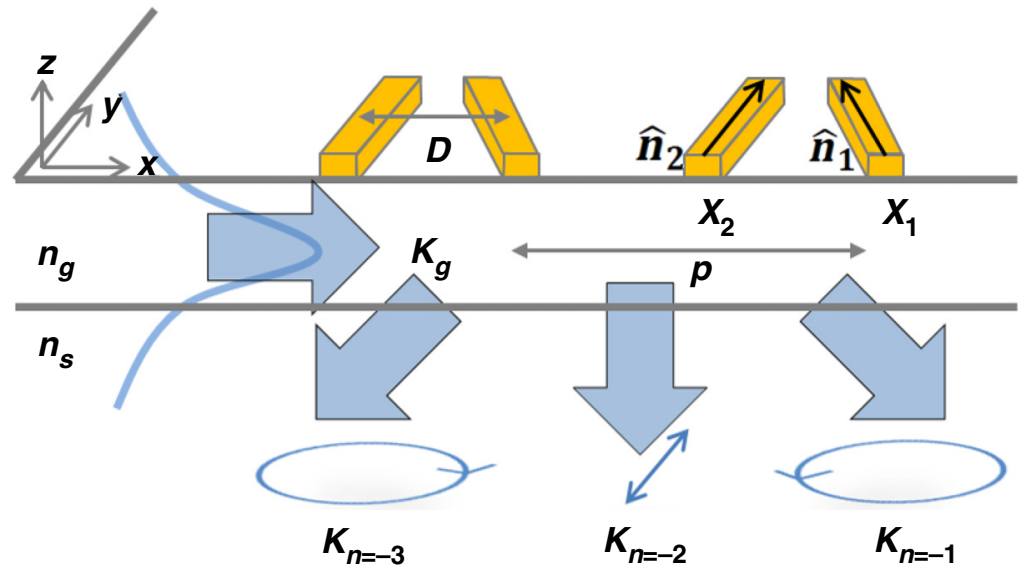

(b)

(b)

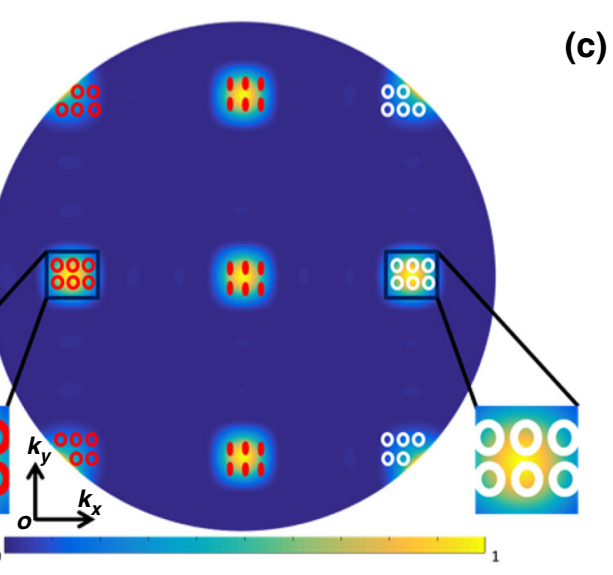

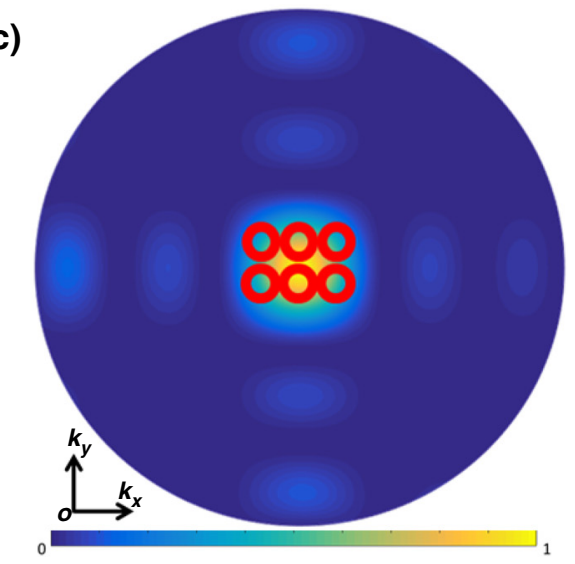

FIG. 4. (a) Sketch of the scattering by $\Lambda$-shaped nanoantennas with an apex angle of $90^{\circ}$. The guided light propagates from the left to right. Three main orders of diffraction light $(n=-1,-2$, and -3 ) along the $O x$ axis show the different LHP, linear, and RHP polarization states, respectively. (b) Simulation of the polarization analysis on the FP. The guided light comes from the left side of the $\Lambda$-shaped antenna array with the same parameters as the experimental sample. Two different directions of elliptical polarization are indicated by red and white. The two insets in the black boxes show the enlarged images of the two main diffraction orders. (c) Simulation of the polarization on the FP for a modified $\Lambda$ shaped antenna array. The array period and the size of antennas are reduced by a factor 2 . The guided light comes from the left side of the $\Lambda$-shaped antenna array. Only one diffraction pattern appears on the FP with circular polarization indicated as red circles. 
which is optimal for collection. The width of the spot can be controlled by the number of repetition on the array; here we keep our $4 \times 4$ nanoantennas. An increase of the number of antennas would induce a reduction of the scattering spot, and thus to a better directivity. The polarization of the scattered light within the diffraction spot can be chosen with the antenna geometry. In order to obtain a circular polarization in the scattered light perpendicular to the surface (order -1 ), the size of each ridge and the distance between two ridges of each $\Lambda$-shaped antenna, approximately divided by two compared with the sample described in Sec. II, are optimal. Fig. 4(c) shows the scattering of a $4 \times 4$ array of nanoantennas whose array period and antenna size are reduced by a factor 2 compared to the previously described design. Only one diffraction order appears in the Fourier plane, and the light is circularly polarized. The handedness changes when excited on the other side of the metasurface.

\section{CONCLUSION}

In conclusion we have demonstrated experimentally that the reverse $\mathrm{SH}$ effect can be used to convert a guided wave into the free-space mode with a polarization depending on the direction of the guided wave and the direction of the free-space mode. For a given guided mode path, the decoupled light can be RHP or LHP depending on the direction selected. By playing on the geometrical parameter of the metasurface, we show that it is also possible to generate a single polarized decoupling of the waveguide, keeping the reversible SH effect. The metasurface-based extraction, controllable with direction and polarization, could be a new building block for multiplexing, chiral sensing, directional light coupling to polarization-controlled switching and manipulation of polarization-encoded photons in photonic integrated circuits aimed at quantum-computing purposes [18]. This general approach can be extended to various frequencies, and could be applied to materials like silicon waveguides or photonic platform [30].

\section{ACKNOWLEDGMENTS}

The Ph.D. grants of Q.J. by the Région Rhône-Alpes and of A.P. by the Ministère de l'enseignement et la recherche, scientifique, are gratefully acknowledged. This work was supported by Agence Nationale de la Recherche (ANR), France, through SINPHONIE Grant No. ANR-12-NANO0019 and PLACORE Grant No. ANR-13-BS10-0007. We thank J.-F. Motte and and G. Julie, from NANOFAB facility in Neel Institute, for the electron-beam lithography used in this work.

\section{APPENDIX: EXPERIMENTAL METHODS}

The waveguide used in the experiments is formed by a $\mathrm{TiO}_{2}$ layer of optical index 1.96, deposited on a glass substrate. The $\mathrm{TiO}_{2}$ film has been fabricated with a solgel method [31] using a dip-coating deposition followed by a $350{ }^{\circ} \mathrm{C}$ thermal annealing. The effective index of the waveguide has been characterized by $M$-line spectroscopy. The measured thickness of the film is $240 \mathrm{~nm}$ and the effective index is 1.83 for the transverse electric (TE) mode and 1.75 for the TM mode.

The $\Lambda$-shaped antennas as well as the coupling gratings have been fabricated by electron-beam lithography followed by a 50-nm thick gold-film deposition and a lift off. The gold is deposited by thermal evaporation under vacuum.

To measure the polarization states on each point of the Fourier plane, the transmitted intensity through the polarizing components is measured on every pixel of the image and for different QWP angles. The Stokes parameters $S_{i=0.3}$ for each pixel are deduced from the dependence of the intensity on the angle of the fast axis of the QWP [32]. The overall intensity is the $S_{0}$ parameter, the polarized light intensity is $\sqrt{S_{1}^{2}+S_{2}^{2}+S_{3}^{2}}$, and the polarization ellipse is defined with the ellipticity and azimuth angles, $\epsilon=$ $\frac{1}{2} \arcsin \left(S_{3}\right)$ and $\theta=\frac{1}{2} \arctan \left(S_{2} / S_{1}\right)$, respectively. From the Stokes parameters, we deduce the overall intensity, the polarization ratio and the polarization ellipticity as described for example in [33].

[1] K. Y. Bliokh, F. J. Rodríguez-Fortuño, F. Nori, and A. V. Zayats, Spin-orbit interactions of light, Nat. Photon. 9, 796 (2015).

[2] L. Marrucci, E. Karimi, S. Slussarenko, B. Piccirillo, E. Santamato, E. Nagali, and F. Sciarrino, Spin-to-orbital conversion of the angular momentum of light and its classical and quantum applications, J. Opt. 13, 064001 (2011).

[3] K. Y. Bliokh, Y. Gorodetski, V. Kleiner, and E. Hasman, Coriolis Effect in Optics: Unified Geometric Phase and Spin-Hall Effect, Phys. Rev. Lett. 101, 030404 (2008).

[4] O. Hosten and P. Kwiat, Observation of the spin Hall effect of light via weak measurements, Science 319, 787 (2008).

[5] J. Ménard, A. E. Mattacchione, M. Betz, and H. M. van Driel, Imaging the spin Hall effect of light inside semiconductors via absorption, Opt. Lett. 34, 2312 (2009).

[6] Z. H. Wang and V. V. Dobrovitski, Time-optimal rotation of a spin 1/2: Application to the NV center spin in diamond, Phys. Rev. B 84, 045303 (2011).

[7] D. O'Connor, P. Ginzburg, F. J. Rodríguez-Fortuño, G. A. Wurtz, and A. V. Zayats, Spin-orbit coupling in surface plasmon scattering by nanostructures, Nat. Commun. 5, 5327 (2014).

[8] J. Petersen, J. Volz, and A. Rauschenbeutel, Chiral nanophotonic waveguide interface based on spin-orbit interaction of light, Science 346, 67 (2014).

[9] F. J. Rodríguez-Fortuño, G. Marino, P. Ginzburg, D. O Connor, A. Martínez, G. Wurtz, and A. V. Zayats, Near-field interference for the unidirectional excitation of electromagnetic guided modes, Science 340, 328 (2013). 
[10] Q. Jiang, A. Pham, M. Berthel, S. Huant, J. Bellessa, C. Genet, and A. Drezet, Directional and singular surface plasmon generation in chiral and achiral nanostructures demonstrated by leakage radiation microscopy, ACS Photonics 3, 1116 (2016).

[11] J. P. B. Mueller and F. Capasso, Asymmetric surface plasmon polariton emission by a dipole emitter near a metal surface, Phys. Rev. B 88, 121410(R) (2013).

[12] P. K. Tien, Light waves in thin films and integrated optics, Appl. Opt. 10, 2395 (1971).

[13] P. Lodhal, S. Mahmoodian, S. Stobbe, P. Schneeweiss, J. Volz, A. Rauschenbeutel, H. Pichler, and P. Zoller, Chiral quantum optics, Nature 541, 473 (2017).

[14] V. Kumar, H. Kaur, and M. Kumar, Narrow-core hollow optical waveguide with nanostructured SOI as ultra-low loss platform for efficient photodetection, Photon. Netw. Commun. 33, 1 (2017).

[15] Z. Chen, G. Wang, X. Wang, and Q. Zhao, Moving toward optoelectronic logic circuits for visible light: A chalcogenide glass single-mode single-polarization optical waveguide switch, Appl. Opt. 56, 1405 (2017).

[16] P. Kozma, F. Kehl, E. Ehrentreich-Förster, C. Stamm, and F. F. Bier, Integrated planar optical waveguide interferometer biosensors: A comparative review, Biosens. Bioelectron. 58, 287 (2014).

[17] F. B. Arango, A. Kwadrin, and A. F. Koenderink, Plasmonic antennas hybridized with dielectric waveguides, ACS Nano 6, 10156 (2012).

[18] F. J. Rodríguez-Fortuño, Daniel Puerto, Amadeu Griol, Laurent Bellieres, J. Martí, and A. Martínez, Sorting linearly polarized photons with a single scatterer, Opt. Lett. 39, 1394 (2014).

[19] F. J. Rodríguez-Fortuño, Daniel Puerto, Amadeu Griol, Laurent Bellieres, J. Martí, and A. Martínez, Universal method for the synthesis of arbitrary polarization states radiated by a nanoantenna, Laser Photon. Rev. 8, L27 (2014).

[20] X. Yin, Z. Ye, J. Rho, Y. Wang, and X. Zhang, Photonic spin Hall effect at metasurfaces, Science 339, 1405 (2013).

[21] P. Rauter, J. Lin, P. Genevet, S. P. Khanna, M. Lachab, A. G. Davies, E. H. Linfield, and F. Capasso, Electrically pumped semiconductor laser with monolithic control of circular polarization, Proc. Natl Acad. Sci. 52, 5623 (2014).
[22] D. Vercruysse, Y. Sonnefraud, N. Verellen, F. B. Fuchs, G. D. Martino, L. Lagae, V. V. Moshchalkov, S. A. Maier, and P. V. Dorpe, Unidirectional side scattering of light by a single-element nanoantenna, Nano Lett. 13, 3843 (2013).

[23] B. Guan, R. P. Scott, C. Qin, N. K. Fontaine, T. Su, C. Ferrari, M. Cappuzzo, F. Klemens, B. Keller, M. Earnshaw, and S. J. B. Yoo, Free-space coherent optical communication with orbital angular, momentum multiplexing/demultiplexing using a hybrid 3D photonic integrated circuit, Opt. Express. 22, 145 (2014).

[24] H. Li, W. Zhou, Y. Liu, X. Dong, C. Zhang, C. Miao, M. Zhang, E. Li, and C. Tang, Preliminary investigation of an SOI-based arrayed waveguide grating demodulation integration microsystem, Sci. Rep. 4, 4848 (2014).

[25] M. L. Brongersma and V. M. Shalaev, The case for plasmonics, Science 328, 440 (2010).

[26] A. Drezet, A. Hohenau, A. L. Stepanov, H. Ditlbacher, B. Steinberger, N. Galler, F. R. Aussenegg, A. Leitner, and J. R. Krenn, How to erase surface plasmon fringes, Appl. Phys. Lett. 89, 091117 (2006).

[27] A. Drezet and C. Genet, Imaging Surface Plasmons: From Leaky Waves to Far-field Radiation, Phys. Rev. Lett. 110, 213901 (2013).

[28] M. Berthel, Q. Jiang, C. Chartrand, J. Bellessa, S. Huant, C. Genet, and A. Drezet, Coherence and aberration effects in surface plasmon polariton imaging, Phys. Rev. E 92, 033202 (2015).

[29] Q. Jiang, A. Pham, S. Huant, J. Bellessa, C. Genet, and A. Drezet, Highly efficient singular surface plasmon generation by achiral apertures, Opt. Lett. 41, 4534 (2016).

[30] F. J. Rodríguez-Fortuño, A. Espinosa-Soria, and A. Martinez, Exploiting metamaterials, plasmonics and nanoantennas concepts in silicon photonics, J. Opt. 18, 123001 (2016).

[31] A. Pillonnet, J. Mugnier, V. Le Bihan, C. Leluyer, G. Ledoux, C. Dujardin, B. Masenelli, D. Nicolas, and P. Mélinon, Rare-earth-based nanoclusters embedded in sol-gel waveguiding thin films, J. Lumin. 119, 560 (2006).

[32] H. G. Berry, G. Gabrielse, and A. E. Livingston, Measurement of the Stokes parameters of light, Appl. Opt. 16, 3200 (1977).

[33] S. Huard, Polarization of Light (Wiley, Paris, 1996). 